\title{
Association between etiopathogenesis of morbidly adherent placenta and adenomyosis
}

\author{
Christopher A. Enakpene ${ }^{1,2}$, Ozgul Muneyyirci-Delale ${ }^{1,2^{*}}$ \\ ${ }^{1}$ Department of Obstetrics and Gynecology, SUNY Downstate Medical Center, New York, USA \\ ${ }^{2}$ Department of Obstetrics and Gynecology, Kings County Hospital Center, New York, USA \\ Email: * ozgul.muneyyirci-delale@downstate.edu
}

Received 25 March 2012; revised 30 April 2012; accepted 11 May 2012

\begin{abstract}
The association between etio-pathogenesis of morbidly adherent placenta (MAP) or placenta cretas and adenomyosis has never been described in medical literature. Contrary to the believe that MAP is due to direct invasion of trophoblastic tissues into the adjacent normal myometrium due to prior uterine surgeries, this article describes how pre-existence of adenomyosis acts as a precursor for the development of placenta cretas. It elucidates how prior uterine traumas such as surgeries, repeated childbirths and endometritis cause endometrial tissues to invade the myometrium as a result of disruption of decidua basalis. The invaded endometrial tissues cause hyperplasia and hypertrophy of surrounding myometrium to form the clinical entity called adenomyosis. The over-expression of bcl-2 oncogene in the endometrium causes inhibition of apoptosis of endometrial cells removing the barrier of trophoblastic tissues to invade the myometrium to form MAP. This hypothesis is based on the similarity of their clinical perspectives, similar pathological description of the two disease entities and their common molecular components. Both diseases increase with age; more in women older than 35 years and also in those with history of previous endometrial traumas such as surgeries, childbirth and endometritis. Both diseases also share common pathological factors and molecular components due to absence of deciduas basalis and over-expression of bcl-2 oncoprotein gene, inhibition of cell apoptosis and failure to find genetic abnormalities such as mutations of K-ras, P53 or LOH. An on-going study looking at uterine specimens from cesarean hysterectomies and pelvic MRI evaluation of patients with retained placentas to prove that preexisting adenomyosis may be a precursor to the development of morbidly adherent placenta is near to conclusion.
\end{abstract}

\footnotetext{
"Corresponding author.
}

Keywords: Morbidly Adherent Placenta (Placenta Accretes); Adenomyosis, Association; Epidemiology; Etio-Pathogenesis and Clinical Perspective

\section{INTRODUCTION}

Adenomyosis is a disorder characterized by the presence of islets within the myometrium that consist of both epithelial and stroma elements of endometrial tissues. It was first described by Rokitansky in 1860 and then clearly defined by Von Reckinghausen in 1896 [1,2]. In 1991, Sathganarayana divided adenomyosis into 3 categories depending on the location of the lesion; basal, deep and surface layers. The minimum myometrial invasion required for diagnosis has been debated, but a depth of 2.5 $\mathrm{mm}$ or half a low-power field (LPF) from the endomyometrial border has been universally accepted in most assessments [3-6]. Any site may be involved, but the posterior wall is affected the most $[7,8]$.

Morbidly adherent placenta is globally referred to as placenta cretas and it is defined as the abnormal adherence either in whole or in part of the placenta to the underlying uterine wall. It occurs when a defect of the decidua basalis results in abnormally invasive placenta implantation. Risk factors include placenta previa, previous cesarean section, advanced maternal age, multiparity and previous uterine curettage. It is classified into 2 broad categories according to depth of myometrial invasion and amount of placenta involvement. A partial or total absence of the deciduas basalis and imperfect development of the fibrinoid layer (Nitabuch layer) results in chorionic villi adherent to superficial myometrium-placenta accreta, or chorionic villi involving myometrium-placenta increta and chorionic villi penetrating full thickness myometrium and involving the serosa-placenta percreta. Classification based on the amount of placental involvement are: focal adherence-when part of the cotyledon is involved, partial adherence-when more than one of the cotyledon is involved and total adherence when whole placenta is involved. 


\section{EPIDEMIOLOGY}

\subsection{Epidemiology of Adenomyosis}

Accurate determination of the prevalence of adenomyosis is difficult because the diagnosis can reliably be made with certainty by microscopic examination of extirpated uterus. It has been estimated to affect $20 \%$ of women, however, it was found in approximately $65 \%$ of women in one study of meticulous histopathological analysis of multiple myometrial sections [9]. Adenomyosis most often co-exists with other organic pelvic pathologies such as leiomyoma, endometriosis and pelvic inflammatory disease. The co-existence of adenomyosis and leiomyoma has been reported in up to $60 \%$ of cases studied. Black women have been reported to have a higher incidence of leiomyomas than Adenomyosis [10]. Adenomyosis is usually found in women 35 - 50 years of age who are multiparous and present with abnormal uterine bleeding, usually hypermnorrhea and secondary dysmenorrheal [11].

\subsection{Epidemiology of Morbidly Adherent Placenta}

Morbidly adherent placenta (MAP) was first reported by Plater in a case of a noble woman; Galla who was delivered on March 25 in the year $88 \mathrm{AD}$ [12]. The placenta was retained and she died. Upon autopsy, the placenta was found to be firmly adherent to the uterine wall above the internal Os. The incidence ranges between 1:2500 to 1:110 [13-15]. The increasing incidence of morbidly adherent placenta is link to the rising cesarean section rate. This is suggested to result from the consequences of failure of reconstitution of the endometrium/decidua basalis after repair of a cesarean incision. Histopathology of the placental bed usually shows that the trophoblasts invade the myometrium without intervening deciduas [16].

\section{ETIO-PATHOGENESIS}

Adenomyosis has been variously described by different experts as "elusive disease" or "déjà vu" disease based on the little knowledge of its pathogenesis. However, the universal agreement is that it is associated with accentuation of the myometrial muscle pattern, multiparity, and endometrial hyperplasia. Common proposed etiologies are hereditary, hormonal influences, trauma, viral transmission, and chronic postpartum endometritis. Some authors suggest that the myometrium is vulnerable to invasion by endometrial hyperplasia. Other authors postulate that the myometrium is vulnerable to invasion by endometrial glands because of the absence of a basal membrane-decidua basalis [17]. A retrospective study by Panganamamula et al. found a link between prior uterine surgery and adenomyosis. The hypothesis is that there is a disruption of the junction of the endometrium and myometrium by any surgical intervention. This results in the endometrial glands growing into the uterine muscle leading to the development of Adenomyosis [18]. However, there is a strong suggestion that adenomyosis does not originate from the basal endometrium based on the variation of cell death repressor activity, bcl-2 gene expression and proliferative marker, ki-67 expression as compared with eutopic Endometrium [19]. Other theories proposed are: invasive tissue growth, developmental origins and uterine inflammation related to childbirth. Risk factors include uterine surgeries such as previous cesarean section, dilatation and curettage, myomectomy, and childbirth.

The ectopic endometrial tissue appears to induce hypertrophy and hyperplasia of the surrounding myometrium, which results in a diffusely enlarged uterus called "globular enlargement". A focal occurrence of adenomyosis can result to nodules; called "adenomyoma" which clinically resemble leiomyoma. The uterus generally does not exceed the size of a pregnant uterus at 12 weeks of gestation.

\section{THE LINK BETWEEN THE ETIOLOGY OF MORBIDLY ADHERENT PLACENTA AND ADENOMYOSIS}

The hypothesis of an association between adenomyosis and future development of second or third trimester retain placenta and morbidly adherent placenta such as placenta accretas can be viewed at three different levels: Clinical, pathological and molecular perspective.

\subsection{Clinical Perspective}

Age and prior uterine surgeries such as cesarean section are independent risk factors for placenta previa-placenta accreta as well as Adenomyosis $[11,20]$. The incidence of both disease entities are increased in women older than 35 years and history of previous uterine surgeries. Disruption of endomyometrial junction by any surgical intervention allows in-growth of endometrial tissue into the myometrium and this creates a pathway for trophoblasts to invade the myometrium during placentation. The association between etiology of morbidly adherent placenta and adenomyosis is based on the hypothesis that pre-existing adenomyosis heralds the development of placenta cretas. Our hypothesis is proposing that patients with suspected adenomyosis based upon its clinical, radiological and hysteroscopy features are more likely to develop placenta cretas. The clinical symptoms of adenomyosis include but not limited to dysmenorrhea, menorrhagia, metrorrhagia, dyspareunia and/or dyschezia. Global shape uterus, hypoechoic or slightly heterogeneous appearance on trans- 
vaginal sonogram and MRI features of widening of the junctional zone of $12 \mathrm{~mm}$ or greater increase the risk of future development of placenta accretas in pregnancy. The presence of irregular endometrium, endometrial defects, hypervascularization, strawberry pattern or cystic hemorrhagic lesions which are hysteroscopic characteristics of possible adenomyosis are also proposed in this hypothesis as risk factors for the development of placenta cretas. Adenomyosis and placenta cretas share common other risk factors such as multiparity, endometritis and previous uterine surgeries such as dilatation and curettage, myomectomy and cesarean section. Any condition that leads to disruption of the endometrium can alter decidualization and placentation during pregnancy which can lead to invagination of placenta tissues.

\subsection{Pathological and Molecular Perspectives}

The apparent similarity between the etio-pathogenesis of morbidly adherent placenta and adenomyosis is based on the common pathological factors such as absence of deciduas basalis and over expression of bcl-2 in both disease entities. There is a possibility that the invading endometrial tissues leaves the decidua basalis devoid of normal endometrium covering leading to invasion of corresponding myometrium by immortal endometrial tissues due to over-expression of the bcl-2 oncoprotein gene, an inhibitor of cell apoptosis. The pathogenesis of placenta cretas is multi-dimensional involving increased but incomplete trophoblast invasion in a background of absent decidua. Placenta cretas result from primary deficiency of decidua, abnormal maternal vascular remodeling and excessive trophoblastic invasion of the myometrium [21].

\subsection{The Role of Bcl-2 Oncoprotein Gene in the Etiology of Morbidly Adherent Placenta and Adenomyosis}

Morbidly adherent placenta is associated with retention of a piece or the whole placenta tissue resulting in uterine sub-involution and delayed secondary postpartum hemorrhage. Increase bcl-2 oncoprotein is associated with inhibition of apoptosis and prolonged cell survival. It is seen mostly in sub-involuted placenta bed as compared with involuted bed. The expression of bcl-2 gene is associated with sub-involution of the utero-placental arteries which inhibit complete resolution of pregnancy induced changes [22]. There is also a decrease cellular apoptosis in adenomyosis and ectopic endometrium due to high bcl-2 gene expression in ectopic endometrium tissues. In a study by Ueki et al, occurrence of adenomyosis was correlated to bcl-2 expression regulated by estrogen and estrogen receptor rather than genetic mutation [23]. Constant expression of bcl-2 with estrogen receptor (ER) and hyper-estrogenic metabolic states promote invagination and spreading of adenomyosis into the myometrium. This hypothesis is also anchored on the failure to find genetic abnormalities such as mutations of K-ras, P53 or LOH [23].

\section{CONCLUSION}

Any patient with retained placental tissues following delivery should be evaluated for prior history of symptoms, radiologic or hysteroscopic features of adenomyosis. Till date, transvaginal sonogram and magnetic resonance imaging are the mainstay of pre-operative diagnosis of adenomyosis with sensitivity of $68 \%$ and $70 \%$ and specificity of $65 \%$ and $86 \%$ respectively [24]. When available, they should be utilized without hesitation. Other diagnostic approaches are pre-operative biopsy, hysterosalpingography and hysteroscopy. The definitive treatment and diagnosis of adenomyosis is hysterectomy and pathological examination of extirpated uterus. However, conservative management such as norethindrone acetate, levonorgestrol impregnated IUD (mirena), estrogen, danazol, GnRH agonists, resection of lesions, hysteroscopic endometrial ablation, uterine artery embolization (UAE) and magnetic resonance guided focused ultrasound surgery (MRgFUS) can be employed [24,25]. Our hypothesis suggesting an association between etiopathogenesis of placenta cretas and pre-existing adenomyosis may be factual rather than a mere proposition. Fertility preserving management of adenomyosis may play a great role in reducing the incidence of placenta cretas. More research study to explore temporal association between the etiology of MAP and adenomyosis is under way. The result of our on-going study on this topic will finalize whether this association is true or not.

\section{REFERENCES}

[1] Rokitansky, K. (1860) Ueber uterus-neubildung. Aerzte zu Wien Ztschr K Gesellsch, 16, 577.

[2] Von Recklinghausen, F. (1896) Die adenomyoma die uterus und tuberwandung ihne abkunft von resten des wolffschen korpeus. August Hirschwald, Berlin.

[3] Atzori, E., Tronci, C. and Sionis, L. (1996) Transvaginal ultrasound in the diagnosis of diffuse adenomyosis. Gynecologic and Obstetric Investigation, 42, 39-41. doi,10.1159/000291887

[4] Vercellini, P., Cortesi, I., De Giorgi, O., et al. (1998) Transvaginal ultrasound versus uterine needle biopsy in the diagnosis of diffuse adenomyosis. Human Reproduction, 13, 2884-2887. doi,10.1093/humrep/13.10.2884

[5] Ascher, S.M., Arnold, L.L., Patt, R.H., et al. (1994) Adenomyosis, prospective comparison of MRI and transvaginalsonography. Radiology, 190, 803-806.

[6] Bazot, M., Darai, E., Rouger, J., et al. (2002) Limitations of transvaginalsonography for diagnsosis of adenomyosis 
with histopathological correlation. Ultrasound in Obstetrics \& Gynecology, 20, 605-611. doi,10.1046/j.1469-0705.2002.00852.x

[7] Benson, R.C. and Sneeded, V.D. (1958) Adenomyosis, a reappraisal of symptomatology. American Journal of $\mathrm{Ob}$ stetrics \& Gynecology, 76, 1044-1061.

[8] Vercellini, P., Ragni, G., Trespidi, L., et al. (1993) Adenomyosis, a déjà vu? Obstetrical \& Gynecological Survey, 48, 789-794. doi,10.1097/00006254-199312000-00004

[9] MCelin, T.W. and Bird, C.C. (1974) Adenomyosis of the uterus. Obstetrics and Gynecology Annual, 3, 425.

[10] Owolabi, T.O. and Strickler, R.C. (1977) Adenomyosis a neglected diagnosis. Obstetrics \& Gynecology, 50, 424427.

[11] Pavlik, R.M. (1995) Adenomyosis, an ignored uterine disease. Nursing Practitioner, 20, 32-43.

[12] Plater, F. (1937) Quoted in Surgery. Obstetrics \& Gynecology, 64, 178.

[13] Gielchinsky, Y., Rojansky, N., Faouliotis, S.T. and Ezra, Y. (2002) Placenta accrete-summary of 10 years, a survey of 310 cases. Placenta, 23, 210-214. doi,10.1053/plac.2001.0764

[14] ACOG committee on obstetric practice (2002) ACOG committee opinion number 266 January 2002, placenta accrete. Obstetrics \& Gynecology, 99, 169-170.

[15] Morken, N.H. and Henriksen, H. (2001) Placenta percreta two cases and review of the literature. European Journal of Obstetrics \& Gynecology and Reproductive Biology, 100, 112-115. doi,10.1016/S0301-2115(01)00422-5

[16] Benirschke, K. and Kaufmann, P. (2000) Pathology of the human placenta. 4th Edition, Springer, New York.

[17] Thompson, J.R. and Davion, R.J. (1986) Adenomyosis of the uterus, an enigma. Journal of the American Medical Association, 78, 305-307.
[18] Panganamamula, U.R., Harmanli, O.H., Isik-Akbay, E.F., et al. (2004) Is prior uterine surgery a risk factor for adenomyosis? Obstetrics \& Gynecology, 104, 1034-1038. doi,10.1097/01.AOG.0000143264.59822.73

[19] Matsumoto, Y., Iwasaka, T., Yamasaki, F. and Sugimori, H. (1999) Apoptosis and ki-67 expression in adenomyotic lesions and the corresponding eutopic endometriosis. $\mathrm{Ob}$ stetrics \& Gynecology, 94, 71-77. doi,10.1016/S0029-7844(99)00279-3

[20] Miller, D.A., Chollet, J.A. and Goodwin, T.M. (1997) Clinical risk factors for placenta previa-placenta accreta. American Journal of Obstetrics \& Gynecology, 177, 210214. doi,10.1016/S0002-9378(97)70463-0

[21] Tantbirojn, P., Crum, C.P. and Parast, M.M. (2008) Pathophysiology of placenta creta, the role of decidua and extravilloustrophoblast. Placenta, 29, 639-645. doi,10.1016/j.placenta.2008.04.008

[22] Kong, T.Y. and Abdul Rahman, H. (1997) Bcl-2 expression delays postpartum involution of pregnancy-induced vascular changes in the human placenta bed. International Journal of Gynecological Pathology, 16, 138-142. doi,10.1097/00004347-199704000-00009

[23] Ueki, K., Kumagai, K., et al. (2004) Expression of apoptosis related protein in adenomyotic uteri treated with danazol and GnRh agonists. International Journal of Gynecological Pathology, 23, 248-258. doi,10.1097/01.pgp.0000130109.80359.57

[24] Rabinovici, J. and Stewart, E.A. (2006) New intervenetional techniques for adenomyosis. Best Practice and Research Clinical Obstetrics and Gynecology, 20, 617-636. doi,10.1016/j.bpobgyn.2006.02.002

[25] Muneyyirci-Delale, O., Chandrareddy, A., Battala, N., et al. (2008) Norethindrone acetate in the medical management of adenomyosis. Obstetrics and Gynecology, 111, 29S. 